\title{
Use of Electronic Health Records to Determine the Impact of Ebola Screening
}

\author{
Julie A. Pavlin¹, Gosia Nowak², Aaron Kite-Powell ${ }^{3}$, Lindsey Beaman ${ }^{1}$ and Timothy \\ Whitman 4 \\ 'Infectious Disease Clinical Research Program and Henry M Jackson Foundation, Uniformed Services University of the Health \\ Sciences, Rockville, MD, USA; ${ }^{2}$ Navy and Marine Corps Public Health Center, Norfolk, VA, USA; ${ }^{3}$ CNTS support to Armed Forces \\ Health Surveillance Center, Silver Spring, MD, USA; ${ }^{W}$ Walter Reed National Military Medical Center, Bethesda, MD, USA
}

\section{Objective}

To present methods of screening chief complaints and laboratory orders to find patients who presented for Ebola Virus Disease (EVD) screening, in order to determine the impact Ebola concern had on the Military Health System (MHS)

\section{Introduction}

Since the largest epidemic of Zaire ebolavirus (EBOV) in recorded history began in Guinea in December 2013, the epidemic has spread to neighboring countries of Liberia and Sierra Leone resulting in an estimation of over 27,000 total cases and over 11,000 deaths to date (1). In response to the widespread social disruption caused by this epidemic in West Africa, President Obama committed approximately 2,000 US service members to deploy to the region and provide humanitarian aid. US military physicians were called upon to evaluate service members returning from West Africa (WA) to rule out EVD. The US military also has a considerable number of beneficiaries who travel to WA to visit friends and relatives placing them at risk for exposure to EBOV and the development of illness upon returning to the US.

We are conducting an expanded surveillance program that employs a standard questionnaire that all providers can use when evaluating a patient at-risk for EVD that will also capture information from historical encounters. The data collected from the questionnaire will be used to assess the frequency with which clinicians are called to evaluate patients for EVD and the resources required. However, we realize that many encounters may not be captured with this method, especially those that are not high enough risk to require consultation with infectious disease (ID) specialists, and are developing ways to screen the Electronic Health Record (EHR) to find additional patients.

\section{Methods}

The Department of Defense's (DoD) Electronic Surveillance System for the Early Notification of Community-based Epidemics (ESSENCE) has the capability to screen the "reason for visit" field in the EHR for any combination of words. Previous to this study, we determined text strings that best captured potential EVD patients. Screening for these words, we can scan the entire EHR in ESSENCE from January 2014 until the termination of the outbreak to determine the number of times patients presented for EVD screening, basic demographic information on their age, gender, location, military status (active duty, retiree, family member), if they presented to the emergency department or outpatient clinic for care, any laboratory tests that were performed and the results and the ICD-9 diagnosis given.

We will also query the EHR for evidence of EBOV laboratory testing to identify all patients who had EBOV-specific tests administered. Currently, only PCR is used by the MHS to detect EBOV. Comprehensive SQL and SAS algorithms for the electronic HL7 data were developed to identify patients with EBOV laboratory tests performed. Algorithms were validated against clinical care records. For these patients, we will determine the laboratory results, whether they were hospitalized, associated diagnoses, and basic demographic information.

\section{Results}

Early in the DoD response to the EVD epidemic, we found 76 patients who presented at 27 military treatment facilities for rule-out EVD from 1 Aug 14 through 17 Oct 14 . The majority (58\%) were post-deployment EVD screening, but many were single concerned individuals. For laboratory testing, from June 2014 through July 2015 , 13 tests were documented on 10 patients at 4 different locations. Upon review of the EHR, 2 "patients" appeared to be testing the lab system with 8 others having suspicion of exposure. No EVD cases have been detected in the MHS to date. The final results of what we are able to obtain from the EHR will be presented.

\section{Conclusions}

We have determined that various elements of the EHR, either through an existing syndromic surveillance system or through a specifically-derived method, can provide useful information on the impact of outbreaks on a health system. For EVD, we found patients that did not come to the attention of the ID specialists due to low risk, but still utilized MHS resources. Use of these methods to find patients of concern could be expanded to other outbreaks.

\section{Keywords}

Ebola; Electronic health record; Screening; Laboratory tests; Chief complaints

\section{References}

1. World Health Organization. Ebola situation report, 12 August 2015 Available at http://apps.who.int/ebola/current-situation/ebolasituation-report-12-august-2015

*Julie A. Pavlin

E-mail: JPavlin@idcrp.org 